\title{
Topical Application of Whitening Agents after Erbium-doped Yttrium Aluminum Garnet Fractional Laser Treatment for Melasma in Asians: A Randomized Controlled Split-face Study
}

\author{
Jeong Eun Kim ${ }^{1}$ \\ Jae Kyung Kim² \\ Joo Yeon $\mathrm{Ko}^{1}$ \\ Young Suck Ro ${ }^{1}$ \\ Sung Eun Chang²
}

1 Department of Dermatology, Hanyang University Hospital, Hanyang University College of Medicine, Seoul, Korea

2 Department of Dermatology, Asan Medical Center, University of Ulsan College of Medicine, Seoul, Korea
Received November 5, 2012

Reviced November 20, 2012

Accepted November 21, 2012

\footnotetext{
Correspondence

Sung Eun Chang

Department of Dermatology and Research Institute of Dermatology, University of Ulsan College of Medicine, Asan Medical Center, 388-1 Pungnap-dong, Songpa-gu,

Seoul 138-736, Korea

Tel: +82-2-3010-3460

Fax: +82-2-486-7831

E-mail: csesnumdagmail.com

(c) Korean Society for Laser Medicine and Surgery

(c) This is an open access article distributed under the terms of the Creative Commons Attribution NonCommercial License (http://creativecommons.org/ licenses/by-nc/3.0) which permits unrestricted noncommercial use, distribution, and reproduction in any medium, provided the original work is properly cited.
}

\begin{abstract}
Background and Objectives
Melasma is a common hyperpigmentation disorder, which can cause refractory cosmetic disfigurement. Although fractional photothermolysis (FP) is an approved treatment for melasma, due to high relapse rates, its clinical efficacy remains controversial, especially in Asians. FP may be more useful for laser-assisted drug delivery. The objective of this study was to assess the efficacy of 2,940 nm erbium-doped yttrium aluminum garnet (Er-YAG) FP for melasma, and to compare laser treatment alone with that using laser-assisted topical whitening agents.
\end{abstract}

\section{Materials and Methods}

The faces of 12 Korean women with melasma were treated with six Er-YAG FP at 2 weeks intervals. One cheek was randomly selected for treatment with topical whitening agent after each laser treatment. The other cheek received treatment with vehicle solution. The modified melasma area severity index (MASI) and subjective physician/patient overall improvement assessments were used for measurement of outcome. These measures were recorded every two weeks during the treatment period and four weeks after the last treatment.

\section{Results}

Er-YAG FP induced clinical improvement after the third or fourth treatment, however, there was a tendency to rebound as the treatment continued. Application of a topical whitening agent after FP yielded significantly better MASI darkness, physician global assessment, and patient self assessment values than laser treatment alone.

\section{Conclusion}

Use of the Er-YAG FP laser initially resulted in improvement of melasma lesions; however, this effect was not sustained. Combination treatment comprising FP followed by application of topical whitening agent may be useful as an adjuvant option for treatment of melasma in Asian subjects, as FP may facilitate the penetration of topical drugs.

\section{Key words}

Asian; Melasma; Fractional photothermolysis; Topical drugs 


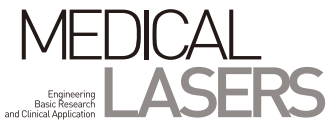

\section{INTRODUCTION}

Melasma is an acquired hyperpigmentation disorder that commonly affects the facial skin. It occurs more frequently in women and can be refractory or recur frequently, particularly in Asians. Numerous treatment options have been developed, including topical bleaching agents such as hydroquinone, retinoic acid, and chemical peels. However, the efficacy of such topical agents is limited: the response to the treatment is often slow or poor, recurrence rates are high, and the rate of penetration into the skin is slow. ${ }^{1,2}$ Moreover, these treatments have the potential to cause skin irritation.

Recently, fractional photothermolysis (FP) was tested for its efficacy against melasma. ${ }^{3-7}$ Several pilot studies showed that FP is effective for treating melasma, and the Food and Drug Administration of the United States has approved a classical FP laser, the Fraxe ${ }^{\circledR} 1,550 \mathrm{~nm}$ erbium-fiber laser, for this purpose. ${ }^{6.7}$ However, most long-term follow-up studies on Asians showed that repetitive FP alone is followed by brief success and then a rebound. ${ }^{5,8}$

Methods to improve the bioavailability of drugs in the deeper skin layers include the biomodulation of drugs and the application of physical modulation techniques, such as treatment with a fractional $\mathrm{CO}_{2}$ laser. A fluorescence study confirmed that fractional $\mathrm{CO}_{2}$ laser treatment facilitates the deep delivery of topical medication into the skin. ${ }^{9}$ However, ablative fractional erbium-doped yttrium aluminum garnet (Er-YAG) laser-assisted drug treatment may be superior to $\mathrm{CO}_{2}$ fractional laser treatment because it may have fewer side effects (such as post-inflammatory hyperpigmentation), while they have a common therapeutic concept of ablative fractional resurfacing for drug delivery.

Thus, we performed an open-label randomized controlled split-face study to evaluate the clinical efficacy and adverse effects of fractional Er-YAG laser both with and without topical whitening agent application for the treatment of melasma in Asian women.

\section{MATERIALS AND METHODS}

\section{Patients}

Twelve Korean women with melasma were enrolled in the study. All had skin type III-IV and their ages ranged from 32 to 52 years (mean, $39.83 \pm 5.80$ years). Patients who were pregnant, lactating, or receiving hormone therapy were excluded. Patients who had used bleaching agents such as hydroquinone cream or who had received laser treatment in the 6 months before enrollment were also excluded. All patients were provided with a detailed description of the purpose and possible outcomes of treatment and gave written informed consent to participate in the study. They also gave their permission for clinical photographs to be taken. This written informed consent was obtained in accordance with our Institutional Review Board-approved protocols, which respect the Declaration of Helsinki guidelines. The study was approved by the Institutional Review Board of the Asan Medical Center (Seoul, Korea).

\section{Treatment}

All treatments were performed 30 minutes after a topical anesthetic cream (EMLA ${ }^{\circledR}$, AstraZeneca, UK) had been applied to the face and left under an occlusive dressing. Fractional ablative photothermolysis treatment was performed using a 2,940 nm erbium-doped yttrium aluminum garnet (Er:YAG) fractional laser (ACTION ${ }^{\top \mathrm{M}}$, Lutronic, Koreal with a 250-us pulse width. The fractional mode of this device divides pulsed light into an array of micro-beams (lens array) that each have a diameter of $250 \mu \mathrm{m}$ and cause vertical microablative columns (density $100 \mathrm{spots} / \mathrm{cm}^{2}$ ) with variable micro-pulse energy (1-14 $\mathrm{mJ}$ ) to form in the epidermis and upper dermis. In addition to the single shot mode, each micro-beam can be shot repeatedly, from twice to ten times, by using the 'multi shot' mode. The user can select a $9 \times 9 \mathrm{~mm}$ or a $12 \times 12$ $\mathrm{mm}$ fractional hand piece tip. The $12 \times 12 \mathrm{~mm}$ fractional hand piece tip was used in the present study. A single pass covers $5 \%$ of the skin. According to unpublished data provided by the manufacturer (derived from a micro-pig model), each single shot with $12 \mathrm{~mJ}$ of energy achieves an approximate penetration depth of $75 \mu \mathrm{m}$.

During each treatment session, the patient underwent one or two passes of full-face FP at energies of $12 \mathrm{~mJ}$. In total, all patients underwent six treatment sessions with 2-week intervals between treatments. After the first laser treatment, one of the patient's cheeks was selected randomly and treated with a topical whitening agent (Dermaheal SB ${ }^{\mathrm{TM}}$; Caregen Co. Ltd, Gyunggi, Korea). The other cheek was treated with vehicle solution. The whitening product consisted of 0.6\% 3-0-ethyl ascorbic acid, $0.4 \%$ arbutin, $0.4 \%$ Glycyrrhiza uralensis root extract, and $0.015 \%$ oligopeptide-34 in water. Ascorbic acid, arbutin, and Glycyrrhiza uralensis root extract are well-known whitening agents, while oligopeptide-34 is a patented agent that inhibits melanin synthesis by decreasing tyrosinase activity and reducing melanosome transfer from melanocytes to keratinocytes (http://www. 
caregen.co.kr/anti-pigmentation05.php). After each of the remaining five Er:YAG laser treatments, the treated cheek was again treated with the topical whitening agent while the control cheek received the vehicle solution.

\section{Evaluation}

After the first treatment, the study subjects returned to the clinic every two weeks for 10 weeks (visit 2-6) and another 4 weeks after the last treatment (visit 7). The study outcomes were measured at every follow-up visit (visit 2-7). The outcome measures used in the present study were the objective melasma severity score (the modified Melasma Area and Severity Index [MASI]], and a subjective assessment of overall improvement made by the physician and the patient.

For the MASI and physician assessments, facial images were taken at all seven visits by using a Robo Skin Analyzer ${ }^{\circledR}$ (CS-50, Inforward, Tokyo, Japan). All images were acquired with the patient standing in the same place under fixed illumination conditions. The Robo Skin Analyzer is a digital image analysis technology system that can quantify the area and severity of hyperpigmentation, wrinkles, and pores. ${ }^{10}$ While MASI values of the right and left cheeks have been used to quantify changes in pigmentation after treatment, the present study used the more objective modified MASI score..11,12

The following formula was used: Modified MASI total score $=0.3 A(f) D(f)+0.3 A(l m) D(l m)+0.3 A(r m) D(r m)+$ $0.1 \mathrm{~A}(\mathrm{c}) \mathrm{D}(\mathrm{c})$ (A: MASI area, D: MASI darkness, f: forehead, Im: left malar, rm: right malar, c: chin)

The total score ranges from 0 to 24. Area and darkness are scored as follows: area of involvement: $0=$ absent, $1 \leq 10 \% ; 2=10-29 \% ; 3=30-49 \% ; 4=50-69 \% ; 5=70-$ $89 \%$; and $6=90-100 \%$. Darkness: $0=$ absent; 1 = slight; $2=$ mild; $3=$ marked; and $4=$ severe. According to this formula, MASI darkness and MASI area were subjected to statistical analysis.

The photographs were also examined by independent
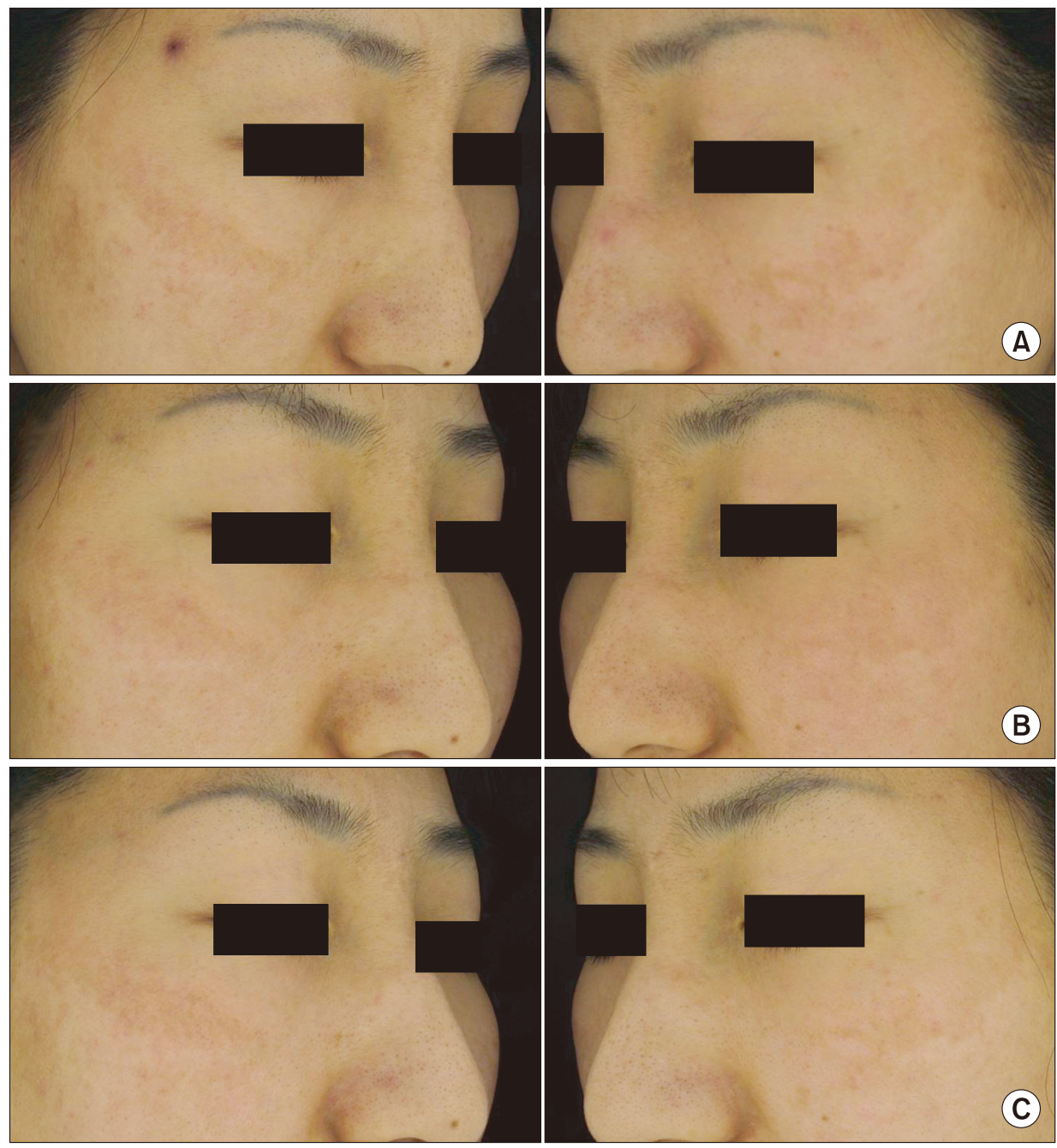

Fig. 1. Serial photographs of a 40-yearold woman with melasma before and after Er-YAG fractional laser treatment. (A) Before treatment. (B) At visit 5 (after fourth treatment). (C) At visit 7 (after sixth treatment). There was an initial improvement followed by melasma rebound after the fifth treatment. However, this was less severe on the laser-assisted topical agent-treated side (right side of this patient) than on the side that treated with the laser alone (left side). 
investigators who evaluated the overall clinical skin improvement using the following 5 -point scale: $0=0 \%$, no improvement; $1=1-25 \%$ improvement; $2=26-50 \%$ improvement; $3=51-75 \%$ improvement; and $4=76$ $100 \%$ improvement. The subjective satisfaction of the patient was also rated using the following 5-point scale: Unsatisfied (0) $=0 \%$, no improvement or worse; Slightly satisfied (1) = 1-25\% improvement; Satisfied (2) $=26-50 \%$ improvement; Very satisfied (3) = 51-75\% improvement; Totally satisfied (4) = 76-100\% improvement.
During the study, all patients were asked to report any adverse symptoms, such as erythema, edema,

Table 1. Baseline homogeneity of outcome measures for each site

\begin{tabular}{lccc}
\hline & Lasertx & Laser-assisted drug tx & \\
\cline { 2 - 3 } & Mean \pm STD & Mean \pm STD & \\
\hline MASI_darkness & $3.00 \pm 0.47$ & $3.10 \pm 0.74$ & 0.6783 \\
MASI_area & $2.90 \pm 0.99$ & $3.00 \pm 0.94$ & 0.5911 \\
\hline
\end{tabular}

Table 2. Longitudinal profile of clinical outcomes

\begin{tabular}{|c|c|c|c|c|}
\hline \multirow{2}{*}{ Outcomes } & \multirow{2}{*}{ Visit } & Laser tx & Laser-assisted drug tx & \multirow{2}{*}{$p$-value } \\
\hline & & LSMean \pm SE & LSMean \pm SE & \\
\hline \multirow[t]{9}{*}{ Physician assessment } & 2 & $1.40 \pm 0.15$ & $1.40 \pm 0.15$ & 0.0042 \\
\hline & 3 & $2.00 \pm 0.16$ & $1.78 \pm 0.16$ & \\
\hline & 4 & $2.51 \pm 0.17$ & $2.13 \pm 0.17$ & \\
\hline & 5 & $2.76 \pm 0.17$ & $2.26 \pm 0.17$ & \\
\hline & 6 & $2.51 \pm 0.17$ & $2.01 \pm 0.17$ & \\
\hline & 7 & $2.01 \pm 0.17$ & $1.51 \pm 0.17$ & \\
\hline & $p(2-4)^{* *}$ & $<0.0001$ & 0.0018 & \\
\hline & $p(2-5)^{* *}$ & $<0.0001$ & 0.0003 & \\
\hline & $p(2-7)^{* *}$ & 0.0092 & 0.6359 & \\
\hline \multirow[t]{9}{*}{ Patient's assessment } & 2 & $1.40 \pm 0.15$ & $1.40 \pm 0.15$ & 0.0033 \\
\hline & 3 & $2.00 \pm 0.16$ & $1.45 \pm 0.16$ & \\
\hline & 4 & $2.38 \pm 0.17$ & $2.01 \pm 0.17$ & \\
\hline & 5 & $2.50 \pm 0.17$ & $2.13 \pm 0.17$ & \\
\hline & 6 & $2.25 \pm 0.17$ & $1.88 \pm 0.17$ & \\
\hline & 7 & $1.75 \pm 0.17$ & $1.38 \pm 0.17$ & \\
\hline & $p(2-4)^{* *}$ & $<0.0001$ & 0.0080 & \\
\hline & $p(2-5)^{* *}$ & $<0.0001$ & 0.0018 & \\
\hline & $p(2-7)^{* *}$ & 0.1211 & 0.9300 & \\
\hline \multirow[t]{10}{*}{ MASI darkness } & 1 & $3.00 \pm 0.19$ & $3.10 \pm 0.19$ & 0.0344 \\
\hline & 2 & $2.40 \pm 0.19$ & $2.90 \pm 0.19$ & \\
\hline & 3 & $2.10 \pm 0.20$ & $2.50 \pm 0.20$ & \\
\hline & 4 & $1.73 \pm 0.21$ & $2.32 \pm 0.21$ & \\
\hline & 5 & $1.85 \pm 0.21$ & $2.19 \pm 0.21$ & \\
\hline & 6 & $2.10 \pm 0.21$ & $2.57 \pm 0.21$ & \\
\hline & 7 & $2.19 \pm 0.22$ & $2.66 \pm 0.22$ & \\
\hline & $p(1-4)^{* *}$ & $<0.0001$ & 0.0020 & \\
\hline & $p(1-5)^{* *}$ & $<0.0001$ & 0.0004 & \\
\hline & $p(1-7)^{* *}$ & 0.0021 & 0.0871 & \\
\hline \multirow[t]{10}{*}{ MASI area } & 1 & $2.90 \pm 0.25$ & $3.00 \pm 0.25$ & 0.3507 \\
\hline & 2 & $2.60 \pm 0.25$ & $2.90 \pm 0.25$ & \\
\hline & 3 & $2.24 \pm 0.25$ & $2.58 \pm 0.25$ & \\
\hline & 4 & $2.19 \pm 0.26$ & $2.50 \pm 0.26$ & \\
\hline & 5 & $2.07 \pm 0.26$ & $2.25 \pm 0.26$ & \\
\hline & 6 & $2.19 \pm 0.26$ & $2.37 \pm 0.26$ & \\
\hline & 7 & $2.25 \pm 0.27$ & $2.86 \pm 0.27$ & \\
\hline & $p(1-4)^{* *}$ & 0.0018 & 0.0236 & \\
\hline & $p(1-5)^{* *}$ & 0.0003 & 0.0009 & \\
\hline & $p(1-7)^{* *}$ & 0.0056 & 0.5432 & \\
\hline
\end{tabular}

Tx, treatment.

*Generalized Linear model: $p$-values for site effects.

**p ("initial visit number"-"follow up visit number"): the value of differences between two time points. 
pain, crusting, infection, post-inflammatory hyper- or hypopigmentation, and scarring.

For statistical analysis, the data obtained at each visit were analyzed using the generalized linear model in the SPSS software (version 12.0; SPSS Inc., Chicago, IL). The data are expressed as the mean \pm standard errors, and $p<$ 0.05 was considered statistically significant.

\section{RESULTS}

\section{Efficacy}

All patients completed the six treatments. The right and left hemifaces of the 12 patients did not differ significantly in terms of average baseline MASI darkness and MASI area values (Table 1). Fig. 1 shows the serial photographs of a representative patient with melasma during the study. As shown in Table 2, the least square mean (LSMean) of MASI darkness decreased on both sides until follow-up visit 4 (after the third treatment). However, the LSMean of

A

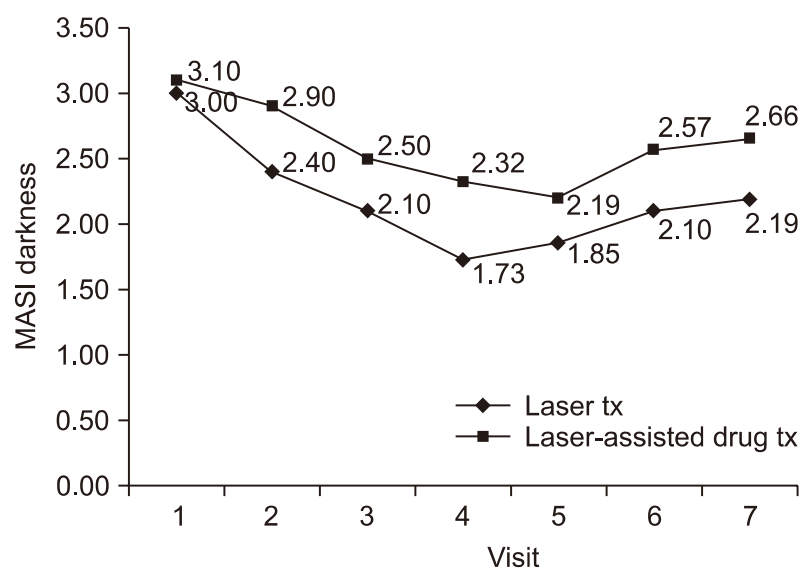

C

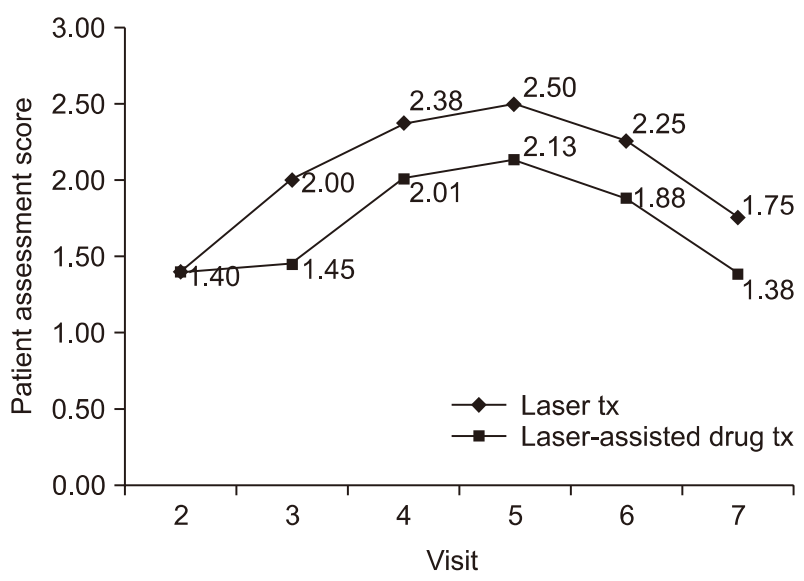

MASI darkness tended to increase after follow-up visits 4-5. Overall, the laser-assisted drug-treated side showed significantly lower LSMean of MASI darkness values than the side that was treated with the laser alone $l p=0.03$, Fig. 2). The LSMeans of MASI area on both sides also dropped until follow-up visit 4-5, after which a similar rebound pattern was observed. However, the laser-alone and combination treatments did not differ significantly in terms of the LSMean of MASI area ( $p=0.35)$.

In terms of clinical improvement, as assessed by the physicians, the melasma lesions on both sides of the face showed clinical improvement compared with the baseline status (Table 2). These improvements were statistically significant until after the fourth treatment, namely, on visit 5 ( $p<0.0001$ for the laser-treated side; $p=0.0003$ for the laser-assisted drug-treated side). After the fifth to sixth treatment, the melasma lesions showed a rebound pattern. However, overall, the side treated with topical whitening agent showed better assessment scores than

\section{B}

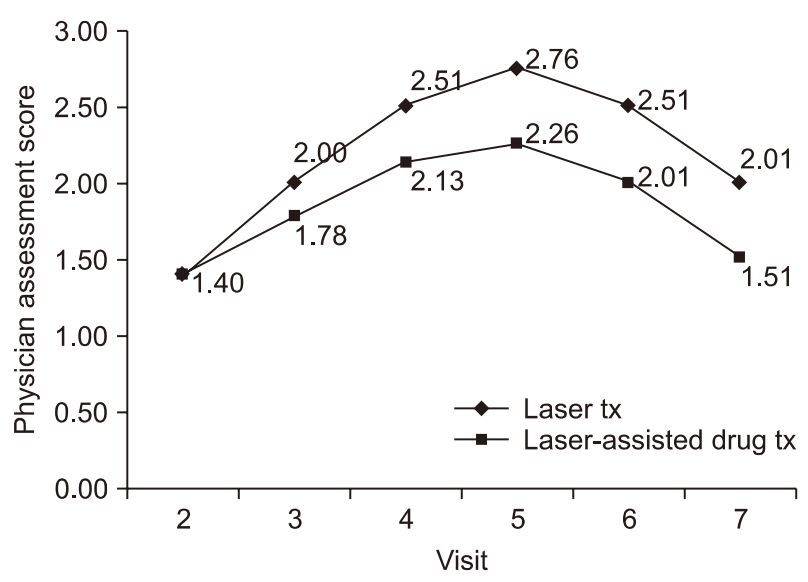

Fig. 2. Clinical outcomes based on standard photographs. (A) MASI darkness. (B) Physician assessment. (C) Patient assessment. 
the side treated with Er-YAG FP alone ( $p=0.0042$ ) (Fig. 2).

The patient self-assessment scores paralleled the physician scores and showed similar statistical results (Table 2, Fig. 2).

\section{Safety}

All patients reported initial reactions to treatment; these reactions consisted of erythema, minimal edema, and microcrust formation on the treated areas. All patients also reported a burning sensation or mild pain; however, this gradually subsided within 2 days. Overall, the erythema and edema were mild and generally resolved within 4 days. The microcrust formation usually lasted for 1 week and then gradually disappeared. Some patients reported an itching sensation and skin dryness, neither of which required additional treatment apart from humectants. There was no evidence of herpes simplex infection, hypopigmentation, or scarring.

\section{DISCUSSION}

The present study examined the efficacy and safety of Er-YAG FP treatment in Asian melasma patients. The outcomes of laser-only treatment and Er-YAG fractional laser-assisted topical whitening agent treatment were also compared. Er-YAG FP initially induced a significant improvement, but a rebound effect occurred as the treatment continued. The application of a topical whitening agent after Er-YAG FP yielded better results than FP treatment alone.

Although several studies show that FP laser treatment is beneficial for melasma patients with a darker skin tone, other studies show that the initial improvement is followed by aggravation of the lesion. ${ }^{3,5,6,8}$ The latter findings are consistent with our own observations.

FP may have a therapeutic effect via three mechanisms. First, a FP laser-induced transport mechanism may remove epidermal and dermal melanin by incorporating it into the micro-epidermal necrotic debris. ${ }^{3,5,13}$ Second, FP may affect melanocytes directly by inducing thermal damage; this could reduce either their capacity to synthesize melanin or their numbers. Lastly, FP may modify the pathological dermal changes associated with melasma, such as increased levels of stem cell factors, altered fibroblasts, or greater vascularity. Recent studies suggest that these dermal changes are pathogenic factors in patients with melasma. ${ }^{14-16}$

Lee et al. ${ }^{5}$ suggested that the micro-thermal zones induced by FP must penetrate the dermis deeply enough to induce dermal remodeling if the incidence of melasma recurrence is to be reduced. Consequently, FP treatment with higher fluence is thought to be more effective for the treatment of dermal type melasma. However, we believe that such a high energy setting may increase the risk of post-inflammatory hyperpigmentation after treatment. Therefore, present study used a drug delivery system namely, the Er-YAG fractional laser with low fluence energy. We postulate that, while the initial improvement was due to the temporary removal of cutaneous melanin, it was followed by a rebound effect because the treatment was repeated several times in relatively short period. This may have led to the accumulation of ablative damage, which in turn meant that subsequent treatments induced greater thermal damage to the dermis, although it may be much less than $\mathrm{CO}_{2}$ laser. Thus, Er-YAG FP treatment alone does not directly inhibit melanocytes and/or the remodeling of the dermis enough to suppress melanogenesis.

In vitro studies showed that chemical agents such as arbutin, retinoids, arbutin, kojic acid, licorice extract, and ascorbic acid can decrease pigment production. ${ }^{17}$ Consequently, these agents are widely used in the prescription, over-the-counter drug, cosmeceutical, and cosmetic fields. However, few of these drugs are clinically effective. Since the therapeutic efficacy of topical drugs is related to their ability to penetrate and distribute throughout the skin layers (thereby reaching the target cells), there is considerable interest in developing novel cutaneous drug delivery systems. ${ }^{18-20}$ One such transdermal drug delivery system may be the ablative resurfacing laser. An in vitro study by Fang et al. ${ }^{21}$ reported that Er:YAG laser treatment increases the permeation of 5-aminolevulinic acid (ALA) more effectively than other delivery systems such as microdermabrasion, iontophoresis, and electroporation. Hsiao et al. ${ }^{22}$ also showed that Er:YAG laser pretreatment increased the transdermal flux of vitamin $\mathrm{C}$ derivatives in mice models. More recently, ablative $\mathrm{CO}_{2}$ fractional resurfacing was shown to facilitate the delivery of a topical drug deep into the skin. ${ }^{9}$ The Er:YAG laser has an advantage over the $\mathrm{CO}_{2}$ laser in that it emits a wavelength of $2,940 \mathrm{~nm}$, which means that incident Er:YAG energy is almost completely absorbed by the water within the epidermis and superficial dermis; this limits the thermal damage to superficial targets and does not cause the underlying thermal damage that can be produced by the longer wavelength $(10,600 \mathrm{~nm})$ of the $\mathrm{CO}_{2}$ laser. ${ }^{22}$ The present study supports the use of the Er:YAG laser for drug delivery as fractional Er:YAG laser resurfacing combined with topical whitening agent application was more 
effective than fractional Er:YAG laser resurfacing alone for treating melasma.

The MASI is the most commonly used outcome measure for melasma treatment. However, its homogeneity component is difficult to assess reliably. Moreover, the area of involvement and darkness are sufficient for the accurate measurement of melasma severity. ${ }^{12}$ Therefore, we used the modified MASI score and physician/patient global assessment to evaluate the efficacy of the treatments. MASI darkness but not MASI area showed that the combination treatment was superior to laser treatment alone in terms of delaying or reducing rebound after treatment.

The main limitations of this study were the small number of patients and the relatively short follow-up periods after the final treatment. However, to the best of our knowledge, this is the first study to compare the effect and time-course of Er-YAG FP laser-assisted topical drug delivery for the treatment of melasma. The melasma lesions in these Asian women improved significantly after the third or fourth treatment, although the lesions recurred as the FP continued. Thus, combination therapy involving the use of topical whitening agents after fractional laser treatment may be an effective adjuvant modality that reduces the relapse of melasma in Asian subjects. Future studies that assess treatment outcomes in larger cohorts of patients with epidermal and dermal melasma are needed. Moreover, the effects of laser parameters, treatment intervals, and long-term maintenance treatment should be evaluated.

\section{REFERENCES}

1. Gupta AK, Gover MD, Nouri K, Taylor S. The treatment of melasma: a review of clinical trials. J Am Acad Dermatol 2006; 55:1048-65.

2. Griffiths CE, Finkel LJ, Ditre CM, Hamilton TA, Ellis CN, Voorhees JJ. Topical tretinoin (retinoic acid) improves melasma. A vehicle-controlled, clinical trial. Br J Dermatol 1993;129:415-21.

3. Katz TM, Glaich AS, Goldberg LH, Firoz BF, Dai T, Friedman PM. Treatment of melasma using fractional photothermolysis: a report of eight cases with long-term follow-up. Dermatol Surg 2010;36:1273-80.

4. Kroon MW, Wind BS, Beek JF, van der Veen JP, NieuweboerKrobotová L, Bos JD, et al. Nonablative 1550-nm fractional laser therapy versus triple topical therapy for the treatment of melasma: a randomized controlled pilot study. J Am Acad Dermatol 2011;64:516-23.

5. Lee HS, Won CH, Lee DH, An JS, Chang HW, Lee JH, et al.
Treatment of melasma in Asian skin using a fractional 1,550-nm laser: an open clinical study. Dermatol Surg 2009;35:1499-504.

6. Rokhsar CK, Fitzpatrick RE. The treatment of melasma with fractional photothermolysis: a pilot study. Dermatol Surg 2005; 31:1645-50.

7. Tannous ZS, Astner S. Utilizing fractional resurfacing in the treatment of therapy-resistant melasma. J Cosmet Laser Ther 2005; 7:39-43.

8. Hong SP, Han SS, Choi SJ, Kim MS, Won CH, Lee MW, et al. Split-face comparative study of $1550 \mathrm{~nm}$ fractional photothermolysis and trichloroacetic acid 15\% chemical peeling for facial melasma in Asian skin. J Cosmet Laser Ther 2012;14:81-6.

9. Haedersdal M, Sakamoto FH, Farinelli WA, Doukas AG, Tam J, Anderson RR. Fractional CO(2) laser-assisted drug delivery. Lasers Surg Med 2010;42:113-22.

10. Kawada A, Kawara S, Oiso N, Endo H, Yoshinaga E, Konishi N, et al. An evaluation of whitening effect of an intense pulsed light source using computer analysis of the video-captured digital image. Arch Dermatol Res 2008;300(Suppl 1):S39-41.

11. Kimbrough-Green CK, Griffiths CE, Finkel LJ, Hamilton TA, Bulengo-Ransby SM, Ellis CN, et al. Topical retinoic acid (tretinoin) for melasma in black patients. A vehicle-controlled clinical trial. Arch Dermatol 1994;130:727-33.

12. Pandya AG, Hynan LS, Bhore R, Riley FC, Guevara IL, Grimes $P$, et al. Reliability assessment and validation of the Melasma Area and Severity Index (MASI) and a new modified MASI scoring method. J Am Acad Dermatol 2011;64:78-83, 83.e1-2.

13. Hantash BM, Bedi VP, Sudireddy V, Struck SK, Herron GS, Chan KF. Laser-induced transepidermal elimination of dermal content by fractional photothermolysis. J Biomed Opt 2006;11: 041115.

14. Kang HY, Hwang JS, Lee JY, Ahn JH, Kim JY, Lee ES, et al. The dermal stem cell factor and c-kit are overexpressed in melasma. Br J Dermatol 2006;154:1094-9.

15. Kang WH, Yoon KH, Lee ES, Kim J, Lee KB, Yim H, et al. Melasma: histopathological characteristics in 56 Korean patients. Br J Dermatol 2002;146:228-37.

16. Kim EH, Kim YC, Lee ES, Kang HY. The vascular characteristics of melasma. J Dermatol Sci 2007;46:111-6.

17. Draelos ZD. Skin lightening preparations and the hydroquinone controversy. Dermatol Ther 2007;20:308-13.

18. Benson HA. Transdermal drug delivery: penetration enhancement techniques. Curr Drug Deliv 2005;2:23-33.

19. Elias PM, Menon GK. Structural and lipid biochemical correlates of the epidermal permeability barrier. Adv Lipid Res 1991;24:1-26.

20. Scheuplein RJ. Percutaneous absorption after twenty-five years: or "old wine in new wineskins". J Invest Dermatol 1976; 67:31-8. 
21. Fang JY, Lee WR, Shen SC, Fang YP, Hu CH. Enhancement of topical 5-aminolaevulinic acid delivery by erbium:YAG laser and microdermabrasion: a comparison with iontophoresis and electroporation. Br J Dermatol 2004;151:132-40.
22. Hsiao CY, Huang CH, Hu S, Ko YS, Sung HC, Huang SY. Skin pretreatment with lasers promotes the transdermal delivery of vitamin C derivatives. Lasers Med Sci 2011;26:369-76. 